\title{
Stellar magnetic fields across the H-R diagram: observational evidence
}

\author{
Svetlana V. Berdyugina ${ }^{1,2}$ \\ ${ }^{1}$ Kiepenheuer Institut für Sonnenphysik, DE-79104 Freiburg, Germany \\ email: sveta@kis.uni-freiburg.de \\ ${ }^{2}$ Institute of Astronomy, ETH Zurich, CH-8093 Zurich, Switzerland
} (EURYI Award Fellow)

\begin{abstract}
This review presents most recent measurements of magnetic fields in various types of stars and substellar objects across the H-R diagram with the emphasis on measurement methods, observational and modeling biases, and the role of magnetic fields in stellar evolution.
\end{abstract}

Keywords. Magnetic fields - molecular processes - polarization - sunspots - stars: activity stars: imaging - stars: spots - stars: evolution - stars: fundamental parameters

\section{Introduction}

An extraterrestrial magnetic field was first discovered in sunspots a century ago (Hale 1908), and it took four decades since then to detect it on a distant star (Babcock 1947). Even though we evidence now a significantly more rapid progress in studying cosmic magnetic fields, it is only around 100-200 stars on which magnetic fields have been detected directly, primarily via the Zeeman effect (ZE) in spectral lines. These fields are believed to be inherited from the interstellar medium during the star formation process and are either fossilized or recycled and amplified by a magnetic dynamo in the stellar interior during subsequent evolutionary stages. Such dichotomy appears to be related to the stellar structure: fossil fields are primary suspects in hot massive stars with outer radiation zones, while dynamo generated fields are attributes of cool stars with outer convection zones (see Fig. 1). However, this simple picture should be taken with a caution as, e.g., little is known how magnetic fields are transported through the stellar interior.

Indirect evidence of magnetic fields on stars operating a magnetic dynamo comes from a multitude of activity phenomena similar to those observed on the Sun, such as spots, plages, chromospheric emission, flares, enhanced X-ray and UV radiation, coronal loops, and coronal mass ejections. Thus, a study of an extensive sample of stars of various activity levels provides key constraints for stellar and solar dynamo models. It was suggested by Skumanich (1972) and confirmed by others that rotation plays a crucial role in the generation of stellar activity and, hence, of magnetic fields, so that cool stars with more rapid rotation show a higher level of magnetic activity. Among single stars these are pre-main-sequence stars ( T Tau-type) and early-age main-sequence stars both of solar type and much cooler red dwarfs. Evolved binary components which are tidally locked at fast rotation by a close companion are also strongly magnetically active (RS CVn-type, BY Dra-type, Algol-like systems as well as FK Com-type which are probably formed from coalesced binaries).

In contrast to the very dynamic appearance of cool stars, presumably fossil magnetic fields on hotter stars appear rather static and topologically uncomplicated, being often low order multipoles or even dipole-like. Most prominently such fields are observed on 
the main sequence Ap-Bp-type stars and white dwarfs, while the topology of magnetic fields on other hot stars remains to be discovered.

The goal of this review is to provide a brief account of the most recent measurements of stellar magnetic fields, analyze underlying assumptions in the interpretation of data, and identify requirements, both observational and theoretical, for obtaining a realistic and relatively complete overview of the magnetic H-R diagram (see Fig. 1). For earlier records see reviews by Mestel \& Landstreet (2005) and Berdyugina (2005).

\section{Zeeman effect in atoms and molecules}

Interaction of an atom or a molecule with an external magnetic field in general leads to splitting of energy levels and, thus, to broadening or even splitting of spectral lines. For relatively weak magnetic fields (when magnetic perturbation $<<$ internal coupling) the splitting is proportional to the magnetic field strength, $B$, and scaled by the effective Landé factor, $g_{\mathrm{eff}}$ :

$$
\Delta \lambda \propto g_{\text {eff }} \lambda^{2} B
$$

It is also proportional to the square of the wavelength. For this reason, successful measurements of magnetic field strengths can be carried out using red or IR lines with large $g_{\text {eff }}$.

Values of $g_{\text {eff }}$ in the cases of pure $L S$-coupling for atoms and Hund's cases (a) and (b) for diatomic molecules are given by simple expressions depending only on quantum numbers of the involved energy levels (Landau \& Lifshits 1991). However, there are examples of atomic transitions for which the $L S$-coupling approximation fails to describe the level structure, and there is almost no molecular transitions well described by the pure Hund's cases due to internal perturbations. In these cases, the effective Landé factor becomes dependent on quantum numbers of involved and perturbing energy levels as well as on coupling constants (e.g., Berdyugina \& Solanki 2002). In case of stronger magnetic fields, when the magnetic perturbation is comparable to or larger than the internal coupling, i.e. in case of the Paschen-Back effect (PBE), $g_{\text {eff }}$ looses its meaning as a constant in Eq. (2.1) as it becomes a function of $B$ (e.g., Berdyugina et al. 2005).

Magnetic diagnostics based on atomic transitions are most often used for measuring stellar magnetic fields, largely because $L S$-coupling violations as well as the $\mathrm{PBE}$ in atoms at stellar magnetic fields (except some Ap stars and white dwarfs) are relatively rare, which significantly simplifies calculations. However, for cooler stars, whose spectra are strongly dominated by molecules, molecular magnetic diagnostics become essential. A significant progress in understanding the molecular Zeeman, Paschen-Back and Hanle effects was achieved in recent years and made it possible to utilize a novel approach for studying stellar and solar magnetism (Berdyugina \& Solanki 2002, Berdyugina et al. 2002, Berdyugina et al. 2003, Berdyugina et al. 2005, Afram et al. 2007, Afram et al. 2008, Asensio Ramos \& Trujillo Bueno 2006, Berdyugina \& Fluri 2004, Shapiro et al. 2007). The PBE appears to be rather common in molecular lines at stellar magnetic fields. It is responsible for Stokes profile asymmetries, net polarization across line profiles, wavelength shifts and polarization sign changes depending on $B$ as well as weakening of main branches and strengthening of satellite and forbidden lines. These pecularities make molecular lines highly sensitive diagnostics despite lower on average effective Landé factors as compared to atomic lines.

A magnetic field measured from line splitting (broadening) of a spectral line (both atomic and molecular) using the Eq. (2.1) approximation represents a mean magnetic field strength averaged over the visible stellar disk irrespective to the field polarity, $\langle|B|\rangle$, 
i.e. assuming that the filling factor of this field, $f$, is unity. In reality, the line profile can contain information on both the field distribution and $f$. However, because of thermal and stellar rotation broadening, this technique is limited to detection of only $\mathrm{kG}$ fields with large filling factors. It was employed in pioneering measurements (Robinson 1980, Saar 1988) and is still useful for surveys of large samples of stars. Reliable measurements require Zeeman splitting larger than or comparable to line widths in the absence of the field.

The most complete way to detect and study stellar magnetic fields is to use the polarimetric technique. The Stokes vector $\vec{I}=(I, Q, U, V)^{T}$ fully describes the polarization state of radiation and contains information on the magnetic field vector, which can be deduced from Stokes measurements by inversion techniques. Realistic estimates can only be obtained by carrying out polarized radiative transfer with detailed calculation of the Muller matrix, which describes a magnetized stellar atmosphere.

However, polarimetry of stars other than the Sun is a rather challenging task and represents a relatively small field of stellar astrophysics. This, on one hand, is due to still very limited instrumental capabilities and, on the other hand, due to disk-integrated observations of the Stokes parameters. The latter results in significant cancellation of the signal from regions of mixed polarity fields and, thus, only large-scale magnetic fields can be detected from disk-integrated polarimetric measurements, which still requires the accuracy of the order $10^{-3}-10^{-4}$. Therefore, most of the current polarimetric measurements were made using only Stokes $V$ parameter, which reveals only a mean longitudinal component of the magnetic field (along the line of sight and with polarity cancellations), $\left\langle B_{z}\right\rangle$, under the assumption that the filling factor $f=1$ for each single-time measurement. The two quantities can be disentangled by analyzing time-series Stokes $V$ measurements (e.g., by ZDI, see Sect. 3).

\section{Zeeman-Doppler Imaging}

Applying an inversion technique to time-series of the four Stokes parameters one can recover the distribution of the temperature and magnetic field vector over the stellar surface, a technique introduced by Semel 1989 and called Zeeman-Doppler Imaging (ZDI). Several numerical codes have been developed for atomic diagnostics: based on the maximum entropy method (Donati et al. 1989, Brown et al. 1991), Tikhonov regularization (Piskunov \& Kochukhov 2002), and principle component analysis (Carroll et al. 2007). Most recently, ZDI employing both atomic and molecular lines has become available (Sennhauser et al. 2008, Berdyugina et al., in preparation).

In practice, however, obtained Zeeman-Doppler stellar images are largely based on measurements of Stokes $I$ and $V$ only. To some extent, the magnetic vector contributes to the line of sight component observed in Stokes $V$ at different rotational phases and different Doppler shifts. For instance, the radial field will dominate the Stokes $V$ near the centre of the stellar disk, while the azimuthal field will be most noticeable in the circular polarization near the stellar limb. This allows for recovering some parts of the magnetic field components from Stokes $V$ observations only. However, the solution is certainly not unique and strongly constrained by assumptions. For instance, in order to limit the solution, a special type of regularization based on spherical harmonic expansion was suggested (Piskunov \& Kochukhov 2002). In this case the solution is forced to take the form of such an expansion which is useful for stars with clearly dominating multipole structures, like e.g. Ap stars. Thus, when interpreting ZDI results obtained only from Stokes $I$ and $V$, one has to take into account that the magnetic field distribution is 
underdetermined for each component and that there might be a cross-talk between different components.

An additional independent constraint on the ZDI solution for cool stars is provided by molecular Stokes profiles. Since molecular lines preferrably form in cooler regions, often associated with magnetic fields, they bear the information on physical conditions in these regions and their location on the stellar surface. Simultaneous inversions of atomic and molecular lines significantly improve the quality of ZDI maps and reduce the cross-talk between magnetic field components (Sennhauser et al. 2008).

\section{Biases in observations and interpretation}

Zeeman signatures in stellar spectra are generally extremely small, with typical relative amplitudes of $0.1 \%$. Detecting them requires measurements of polarization with noise level lower than $10^{-4}$, while the current instrumentation allows for the best relative noise level of $10^{-3}$. Therefore, a multi-line approach for increasing the signal-to-noise ratio of the measured polarization was proposed (Semel 1989, Semel \& Li 1996) and successfully used for detecting stellar circular polarization as an indication of magnetic fields (Least Squares Deconvolution, LSD, Donati et al. 1997).

The underlying assumption in the LSD technique is the weak field approximation (WFA), i.e. one assumes that the magnetic splitting of spectral lines is smaller than their local Doppler broadening. In this case, the Stokes $V$ signal is proportional to the derivative of the intensity profile $I(v)$ in the velocity domain:

$$
V_{i}(v) \propto g_{\mathrm{eff}}(i) \lambda_{i} I_{i}{ }^{\prime}(v),
$$

where $g_{\text {eff }}(i)$ is the effective Landé factor and $\lambda_{i}$ is the wavelength of the $i$ th spectral line. It is assumed further that the local line profiles are self-similar and scale in depth and width with the central depth and wavelength. Finally, the LSD Stokes $V$ profile can be obtained as a sum over many individual lines, i.e. as linear weighted average of line profiles.

A similar approach can be applied to other Stokes parameters with the gain factor in the $\mathrm{S} / \mathrm{N}$ ratio as large as 30 when using more than 2000 line profiles. Drawbacks of this technique are non-linear effects in summation of Stokes profiles and effect of blends (see Sennhauser et al. 2008) as well as diminishing information contents on $T$ and $B$ due to massive averaging. This leads to the fact that LSD profiles cannot be considered anymore as observed but rather processed Zeeman signatures with strong influence of the WFA. The latter is generally not applicable for $|B|>1 \mathrm{kG}$ and $g_{\text {eff }} \sim 1$ and in the case of the $\mathrm{PBE}$. An alternative technique for increasing the $\mathrm{S} / \mathrm{N}$ ratio in spectropolarimetric data is based on the principal component analysis (PCA) which does not directly rely on the WFA (Martínez González et al. 2008).

Usage of LSD profiles for ZDI again involves the WFA combined with the assumption on Gaussian shapes of line profiles (Donati et al. 1989). Furthermore, to overcome a lack of information contained in Stokes $V$ only, a strong constraint on the magnetic field topology in form of a multipole expansion was employed (Piskunov \& Kochukhov 2002, Donati et al. 2006).

Modeling Zeeman-broadened Stokes $I$ profiles often involves template spectra of magnetically active and inactive stars which are weighted by a filling factor (Valenti \& JohnsKrull 2001, Reiners \& Basri 2006):

$$
I_{\lambda}=(1-f) I_{\lambda}(B=0)+f I_{\lambda}(B \neq 0) .
$$


Such an analysis implicitly assumes that (1) the field is concentrated in patches surrounded by field free regions, (2) field is oriented radially in the photosphere, (3) magnetic regions are characterized by a single field strength, and (4) the temperature structure is the same for magnetic and non-magnetic atmosphere Valenti \& Johns-Krull 2001. In this approach, the field strength being determined from the splitting is usually well constrained, but the filling factor depends on the unknown temperature structure of magnetic regions. In the case when the used spectral line becomes stronger in starspots, the filling factor can be overestimated, and vice versa. Also, only stars with strong magnetic fields and large field areas can be studied with this technique.

It is important to emphasize that the assumptions mentioned in this section introduce largely unknown biases in the determined field strengths and distributions. The most obvious improvement is to solve consistently full Stokes radiative transfer equations using stellar atmosphere models. This has been already implemented in the newest ZDI codes and should become the standard approach for interpretation of spectropolarimetric data.

Extracting weak polarization signals with multi-line techniques, such as LSD or PCA, at the level of $10^{-4}$ or smaller relies on the assumption that spectropolarimeters are perfect at such levels, i.e. there are no cross-talks between Stokes parameters. It is however common that a few percent cross-talks can occur between the intensity and polarization as well as between circular and linear polarization. Such cross-talks can be responsible for weak Stokes $V$ signals in stars where linear polarization is due to other processes than magnetic fields, e.g., due to optical pumping and absorptive polarization, which can be of the order of $1 \%$ in Stokes $Q$ and $U$ (Kuhn et al. 2007, Harrington \& Kuhn 2007). In such cases a cross-talk of only $1 \%$ can result in a non-magnetic $10^{-4}$ Stokes $V$ signal, which may confuse a proper interpretation of a magnetic signal. The non-magnetic absorptive polarization appears to be common for stars with circumstellar material, such as Ae-Be stars with disks and winds, AGB stars with envelops, and perhaps upper-main-sequence O-B stars with winds. It is remarkable that Stokes $V$ signals (LSD) detected in spectra of such stars are reported to be very weak are normally interpreted as indication of weak magnetic fields.

\section{Pre-Main-Sequence stars}

\subsection{T Tau stars}

T Tau-type stars are pre-main-sequence stars of about one solar mass at an age of a few million years, still surrounded by disks of gas and dust remaining from their formation. They are believed to be almost fully convective and maintaining vigorous magnetic dynamo. In particular, it is widely accepted that magnetic fields play an important role in the surface and flare activity of T Tau stars as well as in accretion processes and stellar wind phenomena (see reviews by Petrov 2003, Johns-Krull 2009).

Most recent magnetic field measurements in classical T Tauri stars (CTTS) were made by Johns-Krull (2007) from broadening of Zeeman-sensitive Ti II lines in the near IR. It is interesting that the line broadening could not be modeled with one single value of $\langle|B|\rangle$ and required a distribution of fields between $0 \mathrm{kG}$ and $6 \mathrm{kG}$ with different filling factors. When averaged over the visible stellar surface $(f=1)$, the mean field of 12 CTTS was found to be $2.5 \pm 0.3 \mathrm{kG}$ (majority of the sample). It appears that this value exceeds significantly the field strength predicted by simple magnetospheric accretion theory. At the same time, these stars might be underluminous in X-rays as compared to main-sequence stars with the same mean magnetic field strengths. Relatively strong mean fields $(>1 \mathrm{kG})$ detected on all CTTS imply that they are indeed powerful magnetic generators. 
A topology of the magnetic fields on CTTS remains largely unknown. Available ZDI maps of two CTTS (V2129 Oph and BP Tau) are obtained from Stokes $I$ and $V$ LSD profiles only and constrained by a multipole expansion (Donati et al. 2007, 2008a). In the two cases a combination of a relatively weak dipole $(0.35 \mathrm{kG}$ and $1.2 \mathrm{kG})$ and octopole $(1.2 \mathrm{kG}$ and $1.6 \mathrm{kG})$ was found, both slightly tilted with respect to the rotation axis. Accretion spots seem to coincide with the two main magnetic poles at high latitudes and overlap with dark photospheric spots. The average surface field strength in the case of BP Tau is however significantly underestimated compared to that obtained by Johns-Krull (2007).

\subsection{Ae-Be Herbig stars}

Herbig Ae/Be (HAeBe) stars are pre-main-sequence stars of intermediate mass (about 3 to $10 M_{\odot}$ ) embedded in dust-gas circumstellar material similar to CTTS but with hotter photospheres and outer radiation zones. Some HAeBe stars demonstrate activity characteristics normally associated with the presence of chromospheres or coronae, which implies possible presence of magnetic fields and magnetoaccretion.

Several Stokes $V$ surveys with different instruments (Wade et al. 2007, 2009, Hubrig et al. 2009, and references therein) reveal that about $7 \%$ of these stars may possess surface magnetic fields, $\left\langle B_{z}\right\rangle$, in the range of a few hundred Gauss. Note however that polarization signals in embedded stars can have non-magnetic origin too (see Sect. 4). Temporal variations of Stokes $V$ LSD profiles can be described with a dipole field component of about $1 \mathrm{kG}$, which is quite stable over several years. Such a simple and stable topology may imply that magnetic fields are of primordial origin. There are however indications that magnetic fields on Ae stars perhaps correlate with their X-ray luminosity and become very weak or completely disappear when they arrive on the main sequence (Hubrig et al. 2009).

\section{Main-Sequence stars}

\subsection{Massive stars}

Stars in the upper part of the main-sequence with masses exceeding $10 M_{\odot}$ are characterized by strong variable winds, $\mathrm{H}_{\alpha}$ emission variations, chemical peculiarity, and non-thermal radio/X-ray emission. These phenomena can be explained by the presence of magnetic fields in such stars, which still remains a largely unexplored territory. Polarimetric surveys (e.g., Hubrig et al. 2008) in Stokes $V$ found evidence of $\left\langle B_{z}\right\rangle \sim 200 \mathrm{G}$ in only a few stars so far, which may imply that magnetism is not a very common phenomenon among such stars. It is also necessary to verify whether weak circular polarization is not contaminated by a possibly stronger non-magnetic linear polarization due cross-talks (see Sect. 4).

Less massive early B to early $\mathrm{F}$ stars in the upper main sequence host a subclass of chemically peculiar Bp-Ap stars with strongest known magnetic fields among nondegenerate stars, up to $20 \mathrm{kG}$. They comprise only about $5 \%$ of the population and their chemical peculiarity is related to the presence of strong magnetic fields (see a recent review by Mathys 2009). Magnetic fields of Bp-Ap stars cover their whole surface. They have a significant degree of large-scale organization and, in first approximation, their structure is resembling a simple dipole that is inclined to the stellar rotation axis. This can also be clearly seen in ZDI maps (e.g., Kochukhov et al. 2004). Intrinsic variations of these magnetic fields have not been definitely observed so far, which strongly suggests that they might be fossil fields. 


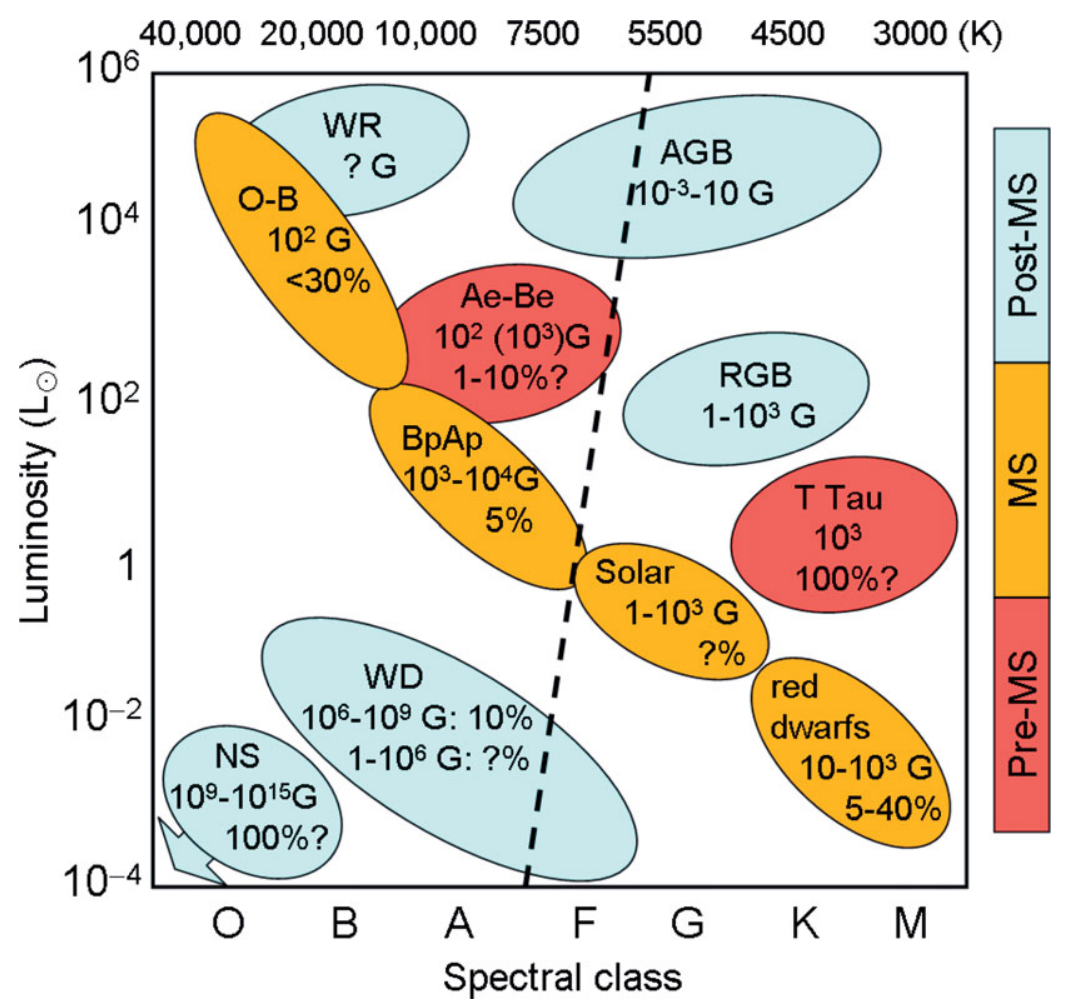

Figure 1. Occurence of magnetic fields across the H-R diagram in pre-MS, MS, and post-MS stars. Percentage indicates the fraction of stars of a given type to have such fields. The dashed line separates stars with convective (on the right) and radiative (on the left) envelops.

\subsection{Solar-type stars}

Solar-type activity on the main sequence is characteristic for stars with subsurface convection zones, roughly from F7 to K2 spectral classes. On the Sun, mean longitudinal field $\left\langle B_{z}\right\rangle$ does not exceed $10 \mathrm{G}$, while local magnetic fields (in plages and sunspots) are of the order of $1-3 \mathrm{kG}$. Unprecedented details of solar magnetic fields are not reviewed here, and the reader should refer to another chapter of these proceedings.

Magnetic activity in solar-type stars declines with age and is closely related to a loss of angular momentum throughout the main-sequence lifetime (see, e.g., Skumanich (1972), Güdel et al. 1997. Thus, young stars exhibit high average levels of activity and rapid rotation, while stars as old as the Sun and older have slower rotation rates and lower activity levels. Also, a change of the magnetic field topology with rotation rate was suggested (Petit et al. 2008), as deduced from ZDI maps of four stars obtained under the multipole expansion approximation from Stokes $V$ LSD profiles. It appears that slower rotators possess mostly poloidal fields, while more rapid counterparts host a large-scale toroidal component. A rotation period of $\sim 12$ days seems to be a threshold for the toroidal magnetic energy to dominate over the poloidal component for shorter periods.

\subsection{Low-mass dwarfs}

Red dwarfs constitute at least $80 \%$ of the stellar population in the Galaxy. Younger stars and binary components exhibit remarkable magnetic activity which is expressed in extremely strong optical flares, starspots, and enhanced UV, X-ray and radio emission, thus, indicating active chromospheres and coronae powered by magnetic fields. Most of 
the knowledge on the latter was deduced from broadening/splitting of line profiles, mainly atomic or $\mathrm{FeH}$ lines, modeled with template stellar spectra which limited the accuracy of the results (e.g., Johns-Krull \& Valenti 1996, Reiners \& Basri 2007). Mean fields of up to $4 \mathrm{kG}$ were found to be common for these stars, with little or no dependence on the mass. The latter appears to be surprising as stars later than M4 are expected to be fully convective and a change of the dynamo mechanism is anticipated in lower mass stars.

Spectropolarimetric surveys of red dwarfs revealed rich Stokes $V$ spectra with many atomic and molecular signatures formed in starspots and chromospheres (Berdyugina et al. 2006, 2008), which provide unique information on the vertical structure of magnetic regions. ZDI maps based on the multipole expansion, weak-field approximation and Stokes $V$ LSD profiles (Donati et al. 2008b, Morin et al. 2008) indicate a possible change in the field topology from largely toroidal configuration in M1 to axisymmetric poloidal components in M4, supposedly due to the onset of a distributed dynamo. However, ZDI based on polarized radiative transfer in many atomic and molecular lines indicates that photosphere temperatures of spotted $\mathrm{M}$ dwarfs correspond to earlier spectral classes and large-scale bipolar regions are seen on both M1 and M4 active red dwarfs (Berdyugina et al., in preparation).

\section{Post-Main-Sequence stars}

A loss of angular momentum during the main sequence stage and beyond leads to overall diminishing of magnetic activity in red giant branch (RGB) and asymptotic giant branch (AGB) stars, except evolved binary components which gain a faster rotation due to tidally locked orbits (RS CVn-type stars).

Single red giants, rotating for some reasons relatively fast, apparently possess mean longitudinal fields $\left\langle B_{z}\right\rangle$ (deduced from Stokes $V$ LSD profiles) comparable to that of old solar-type stars, i.e. 1-10 G (Konstantinova-Antova et al. 2008, 2009). Approximately the same range of field strengths is observed near the photospheres of AGB stars, where SiO masers are formed (Herpin et al. 2006). It reduces with a $1 / r$ law down to $10^{-3}-10^{-1} \mathrm{G}$ at the outskirts of the circumstellar envelope, as measured from $\mathrm{OH}$ masers. Such fields may play a role in shaping circumstellar envelopes in post-AGB objects.

Evolved binary components in RS CVn-type variables are distinguished by their strong chromospheric plages, coronal X-ray and microwave emissions as well as strong flares in the optical, UV, radio and X-ray. Remarkable activity and high luminosity of these stars make them favourite targets for magnetic studies. Earlier ZDI images (based on LSD and the WFA) indicated the presence of a (sub-)kG field with a dominating almost axisymmetric azimuthal component appearing as rings of opposite polarities at higher and lower latitudes (Donati et al. 2003). Recently, a more realistic ZDI approach has lead to detection of a bipolar region with dominant radial field component on the RS CVntype star II Peg (Carroll et al. 2007). The prototype of FK Com-type stars exhibiting activity phenomena very similar to that of RS CVn stars was also confirmed to be a magnetic star with an average longitudinal field $\left\langle B_{z}\right\rangle$ of $250 \mathrm{G}$ at the phase of maximum visibility of cool spots (Korhonen et al. 2009).

Degenerate stellar remnants, such as white dwarfs (WD) and neutron stars (NS), are known to be remarkable magnetic objects, especially in binary systems. Isolated magnetic white dwarfs with fields of $10^{6}-10^{9} \mathrm{G}$, are quite rare, comprising about $5-10 \%$ of all white dwarfs (Wickramasinghe \& Ferrario 2000), while those with kG fields apparently are more frequent, 15-20\% (Jordan et al. 2007). Such a distribution is reminiscent of the occurence of magnetic non-degenerated stars. Observed spectral variations of magnetic white dwarfs on a timescale of hours or days suggest a complex magnetic field distribution 
on their surfaces. In some cases, spot-like magnetic field enhancements superimposed on a weaker dipole magnetic field can be identified. Similar structures are most probably present on the surfaces of neutron stars with field strengths of $10^{9}-10^{15} \mathrm{G}$. Current theory predicts that such structures can be generated from strong subsurface toroidal fields on both white dwarfs and neutron stars.

\section{Conclusions}

The present review indicates that there is a tremendous progress in observations and detections of magnetic fields on various types of stars, thanks to a new generation of spectropolarimetric instruments on large telescopes. At the same time new, significantly more advanced methods for the analysis of spectropolarimetric data based on full Stokes polarized radiative transfer in atomic and molecular lines have been recently developed. They are becoming standard techniques for advancing our knowledge on stellar magnetism and should replace methods with many unrealistic approximations, so that white spots on the H-R diagram are filled with meaningful information.

\section{Acknowledgements}

This work was supported by SNF grant PE002-104552 and the EURYI Award from the ESF.

\section{References}

Afram, N., Berdyugina, S. V., Fluri, D. M., Semel, M., Bianda, M., \& Ramelli, R. 2007, A\& A 473, L1

Afram, N., Berdyugina, S. V., Fluri, D. M., Solanki, S. K., \& Lagg, A. 2008, A $\& A$ 4 482, 387

Asensio Ramos, A. \& Trujillo Bueno, J. 2006, ApJ 636, 548

Babcock, H. W. 1947, ApJ 105, 105

Berdyugina, S. V. 2005, Liv. Rev. Solar Phys. 2, 8

Berdyugina, S. V., Braun, P. A., Fluri, D. M., \& Solanki, S. K. 2005, A\&A 444, 947

Berdyugina, S. V. \& Fluri, D. M. 2004, A\&A 417, 775

Berdyugina, S. V., Fluri, D. M., \& Afram, N., et al. 2008, in: G. van Belle (ed.), 14th Cambridge Workshop on Cool Stars, Stellar Systems, and the Sun, ASP Conf. Ser., vol. 384, p. 175

Berdyugina, S. V., Petit, P., Fluri, D. M., Afram, N., \& Arnaud, J. 2006, in: R. Casini \& B. W. Lites (eds.), Solar Polarization 4, ASP Conf. Ser., vol. 358, p. 381

Berdyugina, S. V. \& Solanki, S. K. 2002, A\&A 385, 701

Berdyugina, S. V., Solanki, S. K., \& Frutiger, C. 2003, A\&A 412, 513

Berdyugina, S. V., Stenflo, J. O., \& Gandorfer, A. 2002, A\&A 388, 1062

Brown, S. F., Donati, J.-F., Rees, D. E., \& Semel, M. 1991, A\&A 250, 463

Carroll, T. A., Kopf, M., Ilyin, I., \& Strassmeier, K. G. 2007, $A N$ 328, 1043

Donati, J.-F., Semel, M., \& Praderie, F. 1989, A\&A 225, 467

Donati, J.-F., Semel, M., Carter, B. D., Rees, D. E., \& Collier Cameron, A. 1997, MNRAS 291, 658

Donati, J.-F., Collier Cameron, A., Semel, M., et al. 2003, MNRAS 345, 1145

Donati J.-F., Howarth I., Jardine M., et al. 2006, MNRAS 370, 629

Donati J.-F., Jardine M., Gregory, S. G., et al. 2007, MNRAS 380, 1297

Donati J.-F. Jardine M., Gregory, S. G., et al. 2008a, MNRAS, 386, 1234

Donati J.-F., Morin, J., Petit, P., et al. 2008b, MNRAS 390, 545

Güdel, M., Guinan, E. F., \& Skinner, S. L. 1997, ApJ 483, 947

Hale, G. E. 1908, ApJ 28, 315

Harrington, D. M. \& Kuhn, J. R. 2007, ApJ 668, L63

Herpin, F., Baudry, A., Thum, C., Morris, D., \& Wiesemeyer, H. 2006, A\& $A$ 450, 667 
Hubrig, S., Schöller, M., Schnerr, R. S., et al. 2008, A\&SA 490, 793

Hubrig, S., Grady, C., Schöller, M., et al. 2009, this proceedings

Johns-Krull, C. M. 2007, ApJ 664, 975

Johns-Krull, C. M. 2009, this proceedings

Johns-Krull, C. M. \& Valenti, J. A. 1996, ApJ 459, L95

Jordan, S., Aznar Cuadrado, R., Napiwotzki, R., Schmid, H. M. \& Solanki, S. K. 2007, AछA 462,1097

Kochukhov, O., Drake, N. A., Piskunov, N., \& de la Reza, R. 2004, A 6 A 424, 935

Konstantinova-Antova, R., Auriére, M., Iliev, I.Kh., et al. 2008, A $\& A 480,475$

Konstantinova-Antova, R., Auriére, M., Schröder, K.-P., \& Petit, P. 2009, this proceedings

Korhonen, H., Hubrig, S., Berdyugina, S. V., et al. 2009, MNRAS, in press

Kuhn, J. R., Berdyugina, S. V., Fluri, D. M., Harrington, D. M., \& Stenflo, J. O. 2007, ApJ $668, \mathrm{~L} 63$

Landau, L. D. \& Lifshits, E. M. 1991, Quantum mechanics: Non-relativistic theory, Oxford, Boston: Butterworth-Heinemann

Martínez González, M. J., Asensio Ramos, A., Carroll, T. A., Kopf, M., Ramírez Vélez, J. C., \& Semel, M. 2008, A\&\&A 486, 637

Mathys, G. 2009, in: S.V. Berdyugina, K.N. Nagendra \& R.Ramelli (eds.), Solar Polarization Workshop 5, ASP Conf. Ser., in press

Mestel, L. \& Landstreet, J. D. 2005, in: R. Wielebinski \& R. Beck (eds.), Cosmic Magnetic Fields, Lect. Notes Phys., vol. 664, p. 183

Morin, J., Donati, J.-F., Petit, P., et al. 2008, MNRAS 390, 567

Petit, P., Dintrans, B., Solanki, S. K., et al. 2008, MNRAS 388, 80

Petrov, P. P. 2003, Astrophysics 46, 506

Piskunov, N. \& Kochukhov, O. 2002, A\&A 381, 736

Reiners, A. \& Basri, G. 2006, ApJ 644, 497

Reiners, A. \& Basri, G. 2007, ApJ 656, 1121

Robinson, R. D., J. 1980, ApJ 239, 961

Saar, S. H. 1988, ApJ 324, 441

Semel, M. 1989, A\&BA 225, 456

Semel, M. \& Li, J. 1996, Sol. Phys. 164, 417

Sennhauser, C., Berdyugina S. V. \& Fluri, D. M. 2008, in: S. V. Berdyugina, K. N. Nagendra \& R.Ramelli (eds.), Solar Polarization Workshop 5, ASP Conf. Ser., in press

Shapiro, A. I., Berdyugina, S. V., Fluri, D. M., \& Stenflo, J. O. 2007, A 6 A 475, 349

Skumanich, A. 1072, ApJ 171, 565

Valenti, J. A. \& Johns-Krull, C. M. 2001, in: G. Mathys, S. K. Solanki, D. T. Wickramasinghe (eds.), Magnetic Fields Across the Hertzsprung-Russell Diagram, ASP Conf. Ser. (ASP: San Francisco), vol. 248, p. 179

Wade, G. A., Bagnulo, S., Drouin, D., Landstreet, J. D., \& Monin, D. 2007, MNRAS 376, 1145

Wade, G. A., Alecian, E., Grunhut, J., et al. 2009, in: P. Bastien \& N. Manset (eds.), ASP Conf. Ser., in press

Wickramasinghe, D. T. \& Ferrario, L. 2000, PASP 112, 873 